\title{
MicroRNA-140-5p aggravates hypertension and oxidative stress of atherosclerosis via targeting Nrf2 and Sirt2
}

\author{
QING-QUAN LIU ${ }^{1 *}$, KE REN $^{1 *}$, SU-HONG LIU ${ }^{1}$, WEI-MIN LI $^{2}$, CHANG-JUN HUANG ${ }^{1}$ and XIU-HUI YANG ${ }^{1}$ \\ ${ }^{1}$ Department of General Surgery II, Central Hospital of Luohe, Luohe, Henan 462000; \\ ${ }^{2}$ Department of Vascular Surgery, The Ninth People's Hospital, Shanghai Jiaotong University \\ School of Medicine, Shanghai 200011, P.R. China
}

Received April 7, 2018; Accepted October 19, 2018

DOI: $10.3892 / \mathrm{ijmm} .2018 .3996$

\begin{abstract}
In the present study, the function of microRNA (miR)-140-5p on oxidative stress in mice with atherosclerosis was investigated. A reverse transcription-quantitative polymerase chain reaction assay was used to determine the expression of miR-140-5p. Oxidative stress kits and reactive oxygen species (ROS) kits were used to analyze alterations in oxidative stress and ROS levels. The alterations in protein expression were determined using western blot analysis and an immunofluorescence assay. miR-140-5p expression was increased in mice with atherosclerosis with hypertension. Consistently, miR-140-5p expression was also increased in mice with atherosclerosis. Upregulation of miR-140-5p increased oxidative stress and ROS levels by suppressing the protein expression of nuclear factor erythroid 2-related factor 2 (Nrf2), sirtuin 2 (Sirt2), Kelch-like enoyl-CoA hydratase-associated protein 1 (Keap1) and heme oxygenase 1 (HO-1) in vitro. By contrast, downregulation of miR-140-5p decreased oxidative stress and ROS levels by activating the protein expression of Nrf2, Sirt2, Keap1 and HO-1 in vitro. Sirt2 agonist or Nrf2 agonist inhibited the effects of miR-140-5p on oxidative stress in vitro. Collectively, these results suggested that miR-140-5p aggravated hypertension and oxidative stress of mice with atherosclerosis by targeting Nrf2 and Sirt2.
\end{abstract}

\section{Introduction}

Cardiovascular disease is currently the leading cause of human mortality worldwide and represents a major detriment to human health (1). It is widely acknowledged that the

Correspondence to: Dr Xiu-Hui Yang, Department of General Surgery II, Central Hospital of Luohe, 54 Renmin Road East, Shaoling, Luohe, Henan 462000, P.R. China

E-mail: yangxiuhui2008@163.com

${ }^{*}$ Contributed equally

Key words: microRNA-140-5p, atherosclerosis, nuclear factor erythroid 2-related factor 2 , sirtuin 2 , oxidative stress, reactive oxygen species pathological process of Atherosclerosis (AS) is one of the main causes of the occurrence, evolution and deterioration of AS (1). Further investigation of biomarkers that are directly associated with the pathogenesis of AS and acute myocardial infarction is required. In this way, a more accurate basis for the diagnosis of AS, the evaluation of disease severity and prognostic prediction may be provided (2).

Oxidative stress is associated with the pathogenesis of multiple cardiovascular diseases. Oxidative stress serves a key function in the pathophysiology of diseases including hypertension and AS by causing endothelial dysfunction (3). Clinical studies have identified that risk factors leading to AS exert adverse effects on the number and function of endothelial progenitor cells, limiting the capability for angiogenesis in patients (4). Oxidative stress has been identified to be associated with the pathogenesis of AS (4).

Nuclear factor erythroid 2-related factor 2 (Nrf2) is an important transcription factor of the antioxidant stress pathway (5). Under normal physiological conditions, $\mathrm{Nrf} 2$ is stably bound to Kelch-like enoyl-CoA hydratase-associated protein 1 (Keap1) and is stabilized in the cytoplasm by Keap1 (5). The bound Nrf2 cannot translocate into the nucleus, and neither is it able to exert its unique transcriptional activity (6). Under stress, Keap1 can be decoupled from Nrf2, and unbound Nrf2 is then translocated into the nucleus and bind to the antioxidant element heme oxygenase 1 (HO-1) (6). This process, to a certain extent, enhances the gene expression of its downstream enzymes (5). Among them, the most typical include ubiquitin, phase II detoxification enzymes, antioxidant proteins and proteasomes, expression of which further increases the ability of cells to resist oxidative stress (7).

Cardiovascular disease is a major group of diseases that are detrimental to human health. According to its pathogenesis, cardiovascular disease may be discussed and analyzed with regard to various aspects, including oxidative stress, inflammatory reaction, apoptosis and autophagy, myocardial ischemia-reperfusion and aging, and energy restrictions (8). However, it has been identified that sirtuin family members are associated with the occurrence and development of pathogenesis (8). In addition, sirtuin family members exhibited a marked association with risk factors of cardiovascular diseases, including glucose and lipid metabolism (9). The sirtuin family is a group of regulators of silencing information, which 
includes seven members, namely Sirt1-Sirt7 (10). However, it has been demonstrated that Sirt2 serves a similar or opposite function in cardiovascular disease and its pathogenesis, and the biological function of Sirt2 in oxidative stress has been reported recently (10). Owing to the increased levels of hydrogen peroxide, oxidative stress increases the expression of Sirt2 in NIH3T3 cells (10).

MicroRNAs (miRNAs) are a class of endogenous small non-coding RNAs widely distributed in eukaryotic organisms, which specifically recognize target mRNAs (11). miRNAs serve a regulatory function at the post-transcriptional level by modulating the translation and degradation of target gene mRNAs (12). There are 2,000 types of miRNA in the human genome, which regulate $\sim 30 \%$ of human gene expression (12). miRNAs have been identified to be markedly stable in the serum, plasma, urine and other bodily fluids through the protection of endogenous RNases. Previous studies have identified the existence of various specific miRNAs in the cardiovascular system $(12,13)$. miRNAs have been identified to serve an important function in multiple pathophysiological processes of the cardiovascular system, including regulation of cardiac development, modulation of cardiac function and involvement in myocardial cell apoptosis $(12,13)$. In addition, miRNAs have also been identified to be involved in the major pathological process of coronary AS (13). These results indicate that miRNAs may be novel biomarkers for the early diagnosis, treatment and prognostic prediction of cardiovascular diseases (14). They may be used to further evaluate the degree of AS. Therefore, we hypothesize that, in the future, manipulation of miRNA expression may be used to suppress the progression of coronary AS, which provides a novel approach for the treatment of cardiovascular diseases (14). In the present study, the function of microRNA (miR)-140-5p on hypertension and oxidative stress of AS was investigated.

\section{Materials and methods}

Animals and ethical approval. Wild-type (Wt; male C57BL/6 mice, $\mathrm{n}=6)$ and apolipoprotein $\mathrm{E}(\mathrm{ApoE})^{-/-}$mice (male, $\left.\mathrm{n}=6\right)$ were purchased from Beijing Vital River Laboratory Animal Technology Co., Ltd. (Beijing, China). Mice were housed at $22-23^{\circ} \mathrm{C}, 55-60 \%$ humidity, 12 -h light $/ 12$-h dark cycle, with free access to food and water. Mice were switched to a high-fat $\operatorname{diet}(21 \%$ fat and $0.15 \%$ cholesterol) for 16 weeks. The study protocols were approved by the Ethics Committee of The Ninth People's Hospital (Shanghai Jiaotong University School of Medicine, Shanghai, China). Wt C57BL/6 mice were the control group and $\mathrm{ApoE}^{-/}$mice were the AS group.

Microarray assay. RNA (500 ng) was hybridized to Affymetrix HG-U133 Plus 2.0 GeneChip arrays (containing 54,675 probe sets). Data were analyzed using Ingenuity Pathway Analysis (Qiagen, Inc., Valencia, CA, USA).

Reverse transcription-quantitative polymerase chain reaction (RT-qPCR) assay. RNA samples were obtained from blood vessel tissue samples or cells using TransZol ${ }^{\circledR}$ (Invitrogen; Thermo Fisher Scientific, Inc., Waltham, MA, USA), according to the manufacturer's protocol. cDNA was synthesized from $1 \mu \mathrm{g}$ total RNA using a ReverTra Ace ${ }^{\circledR}$ qPCR RT master mix kit
(Toyobo Life Science, Osaka, Japan), according to the manufacturer's protocol. qPCR was performed using SYBR-Green PCR master mix-plus (Toyobo Life Sciences) on a 7500 real-time PCR system (Applied Biosystems; Thermo Fisher Scientific, Inc.). Thermocycling conditions were: $94^{\circ} \mathrm{C}$ for $10 \mathrm{~min}$, and 40 cycles of $94^{\circ} \mathrm{C}$ for $30 \mathrm{sec}, 57^{\circ} \mathrm{C}$ for $30 \mathrm{sec}$ and $72^{\circ} \mathrm{C}$ for 30 sec. Primers for miR-140-5p were 5'-CAGUGGUUUUACCCU AUGGUAG-3' (forward) and 5'-ACCACAGGGUAGAACACG GAC-3' (reverse); and for U6, used as a reference gene, were 5'-GCTTCGGCAGCACATATACTAAAAT-3' (forward) and 5'-CGCTTCACGAATTTGCGTGTCAT-3' (reverse). The relative gene expression was analyzed using the $2^{-\Delta \Delta C q}$ method (15).

Hematoxylin and eosin $(H \& E)$ staining. Following induction of the AS model, aorta tissue was collected and washed with PBS. Tissue samples were fixed with $4 \%$ paraformaldehyde for $24 \mathrm{~h}$ and embedded in paraffin, and the 5- $\mu \mathrm{m}$-thick sections were stained with $\mathrm{H} \& \mathrm{E}$ for $15 \mathrm{~min}$ at room temperature. Images of cells were captured under fluorescence microscopy (magnification, x100).

Cell culture and transfection. Human umbilical vein endothelial cells (HUVECs) were obtained from the Shanghai Cell Bank of the Chinese Academy of Sciences (Shanghai, China) and were maintained in Dulbecco's modified Eagle's medium supplemented with $10 \%$ fetal bovine serum in a humidified atmosphere containing $5 \% \mathrm{CO}_{2}$ at $37^{\circ} \mathrm{C}$. miR-140-5p, anti-miR-140-5p and negative mimic were purchased from Guangzhou RiboBio Co., Ltd. (Guangzhou, China) and transfected into cells using Lipofectamine ${ }^{\circledR} 2000$ (Invitrogen; Thermo Fisher Scientific, Inc.). Following transfection for $24 \mathrm{~h}$, cells were stimulated with $70 \mu \mathrm{g} / \mathrm{ml}$ oxidized low-density lipoprotein (ox-LDL) for $24 \mathrm{~h}$. Next, following transfection for $4 \mathrm{~h}$, cells were treated with $10 \mu \mathrm{M}$ SRT 1720 hydrochloride (Sirt2 agonist; MedChemExpress, Monmouth Junction, NJ, USA), $1 \mu \mathrm{M}$ NK-252 (Nrf2 agonist; MedChemExpress) and $5 \mu \mathrm{M}$ Troxerutin [reactive oxygen species (ROS) inhibitor; MedChemExpress] for $20 \mathrm{~h}$, and cells were stimulated with $70 \mu \mathrm{g} / \mathrm{ml}$ ox-LDL for $24 \mathrm{~h}$.

Oxidative stress and ROS measurement. Following induction of the AS model for 16 weeks, mice were sacrificed by decapitation following anesthesia with $35 \mathrm{mg} / \mathrm{kg}$ pentobarbital sodium, and serum was collected at $2,000 \mathrm{x}$ g for $10 \mathrm{~min}$ at $4^{\circ} \mathrm{C}$. Serum was used to determine malondialdehyde (MDA), superoxide dismutase (SOD), glutathione (GSH) and glutathione peroxidase (GSH-Px) levels using ELISA kits. Next, cells were collected at $1,000 \mathrm{x}$ g for $10 \mathrm{~min}$ at $4^{\circ} \mathrm{C}$ and ROS (cat. no. E004), MDA (cat. no. A003-1), SOD (cat. no. A001-1-1), GSH (cat. no. A006-2) and GSH-Px (cat. no. A005) levels were determined using ELISA kits (Nanjing Jiancheng Biological Engineering Institute, Nanjing, China). In addition, ROS levels were determined in cells using $2^{\prime}, 7^{\prime}$-dichlorodihydrofluorescein diacetate $(10 \mathrm{mM})$ for $30 \mathrm{~min}$ at room temperature and cells were observed using fluorescence microscopy (magnification, x200).

Dual-luciferase reporter assay. The plasmids containing the wild-type Nrf2, wild-type Sirt2, mutant Nrf2, mutant Sirt2 and miR-140-5p mimic were purchased from Guangzhou RiboBio Co., Ltd. These were co-transfected into HUVECs 


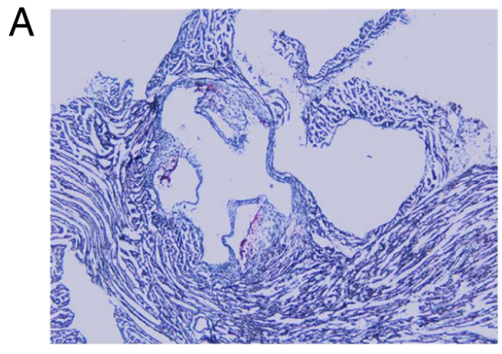

Control

B
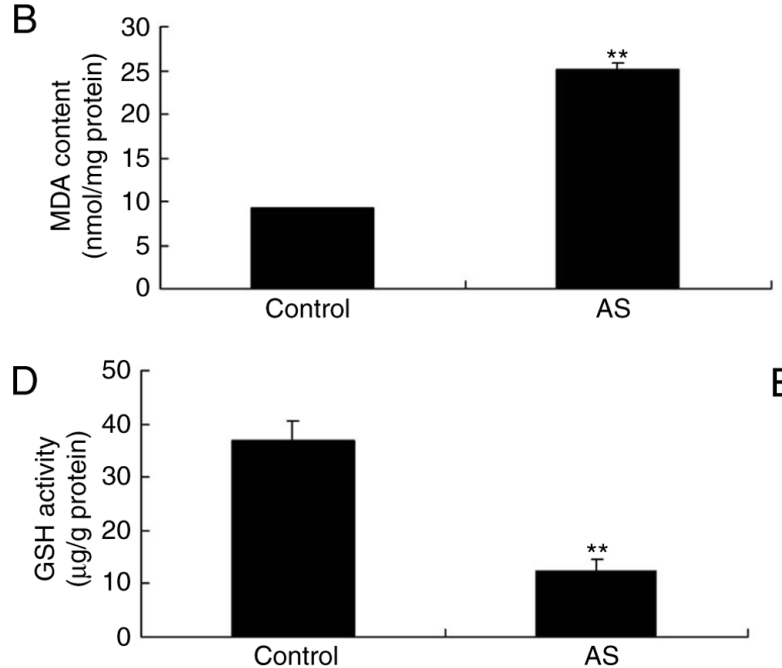

F Control AS

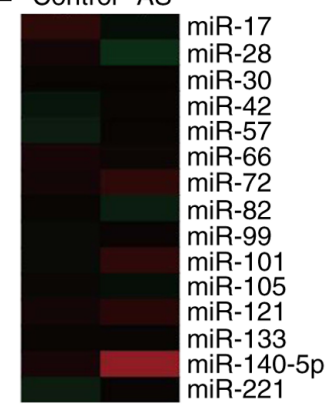

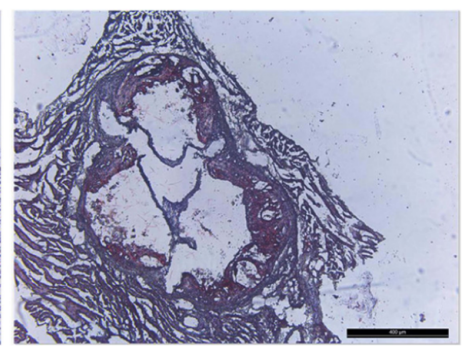

AS

C

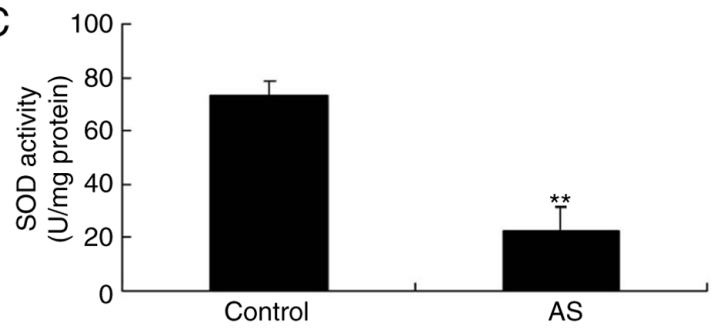

E

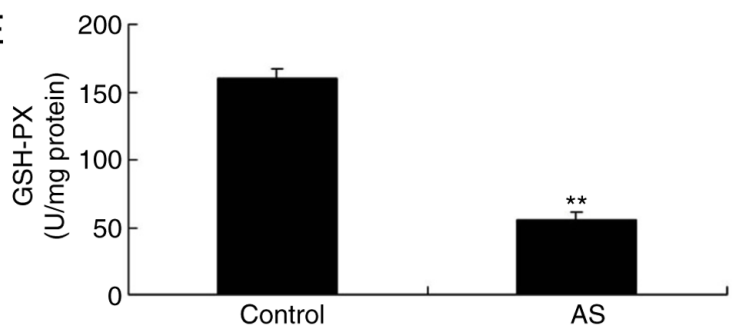

G

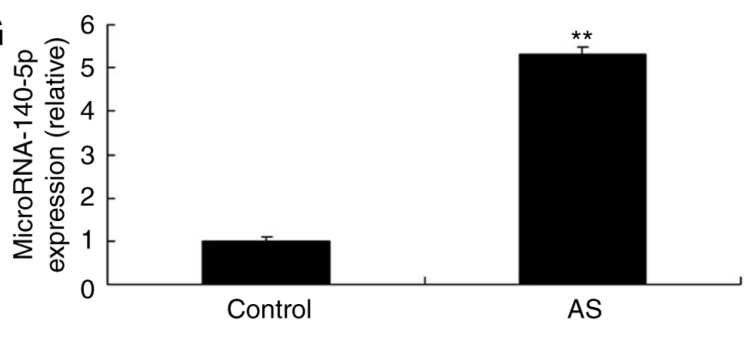

Figure 1. miR-140-5p expression in mice with AS. (A) Hematoxylin and eosin staining of blood vessels. Determination of (B) MDA, (C) SOD, (D) GSH and (E) GSH-Px levels. (F) Gene chip assay. (G) Quantitative polymerase chain reaction analysis of alterations in miR-140-5p. ${ }^{* *} \mathrm{P}<0.01$ vs. control (wild-type mice). miR, microRNA; AS, atherosclerosis; MDA, malondialdehyde; SOD, superoxide dismutase; GSH, glutathione; GSH-Px, glutathione peroxidase.

using Lipofectamine $200024 \mathrm{~h}$ after transfection. Luciferase activity was determined using a Dual-Luciferase Reporter assay kit (Promega Corporation, Madison, WI, USA).

Western blot analysis. Cells were homogenized using radioimmunoprecipitation assay lysis buffer and the protein concentrations of the samples were determined using a bicinchoninic acid protein assay kit (Bio-Rad Laboratories, Inc. Hercules, CA, USA). The proteins (50 $\mu \mathrm{g} /$ lane) were separated by SDS-PAGE (10\% gel) and transferred onto polyvinylidene difluoride membranes (EMD Millipore, Billerica, MA, USA). Membranes were blocked with 5\% non-fat milk in Tris-buffered saline for $2 \mathrm{~h}$ at room temperature and were incubated with primary antibodies: Anti-Nrf2 (cat. no. 12721; 1:2,000; Cell Signaling Technology, Inc., Danvers, MA, USA), anti-Sirt2 (cat. no. 12672; 1:2,000; Cell Signaling Technology, Inc.) and anti-GAPDH (cat. no. 5174; 1:50,000; Cell Signaling
Technology, Inc.) at $4^{\circ} \mathrm{C}$ overnight. Membranes were washed with Tris-buffered saline with $0.1 \%$ Tween-20 (TBST) for $20 \mathrm{~min}$ and incubated with horseradish peroxidaseconjugated anti-rabbit immunoglobulin (GE Healthcare, Chicago, IL, USA) at $37^{\circ} \mathrm{C}$ for $1 \mathrm{~h}$. Membranes were developed with enhanced chemiluminescence substrate solution (GE Healthcare) and analyzed using Image Lab (version 3.0; Bio-Rad Laboratories, Inc.).

Immunofluorescence assay. Cells were washed with PBS and fixed with $4 \%$ paraformaldehyde for $15 \mathrm{~min}$ at room temperature. Cells were blocked with $5 \%$ bovine serum albumin (Beyotime Institute of Biotechnology, Haimen, China) in TBST and $0.25 \%$ Triton X-100 for $1 \mathrm{~h}$ at room temperature. Cells were incubated with anti-Nrf2 and anti-Sirt 2 primary antibodies at $4^{\circ} \mathrm{C}$ overnight. Cells were washed with goat anti-rabbit immunoglobulin G-Cruz Fluor ${ }^{\circledR} 488$ or 555 (1:100; 


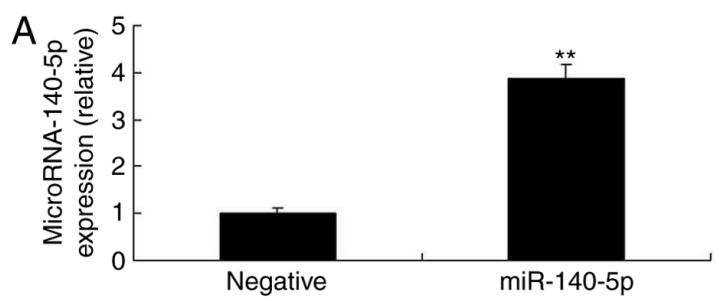

B Negative $\underset{5 p}{\operatorname{miR}-140}$
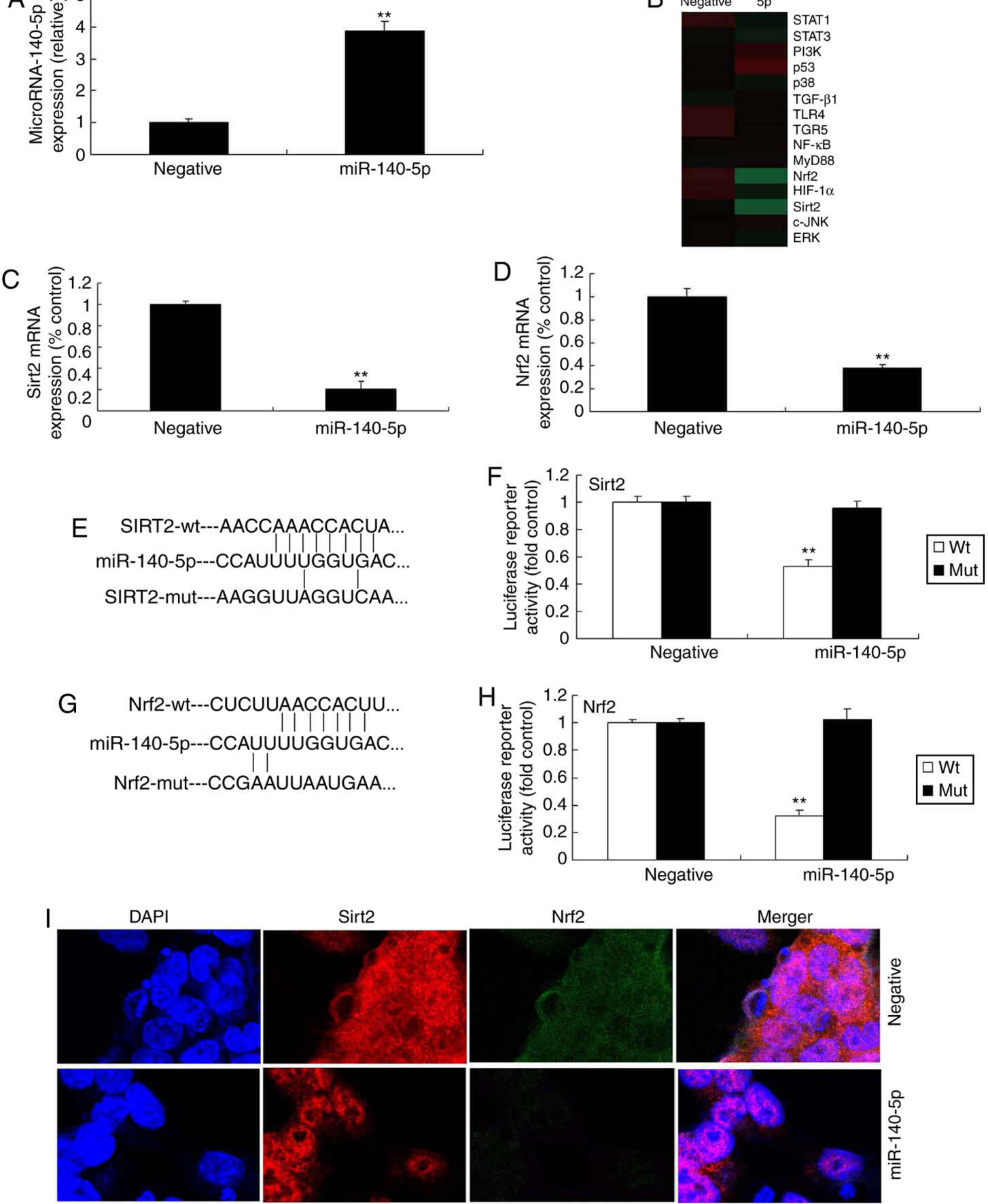

Figure 2. miR-140-5p regulates Nrf2 and Sirt2 expression. (A) qPCR analysis of miR-140-5p. (B) Gene chip assay for signaling pathway members. qPCR analysis for (C) Nrf2 and (D) Sirt2 mRNA expression. (E) miR-140-5p targeted Sirt2 sequence. (F) Dual-luciferase reporter assay for Sirt2. (G) miR-140-5p targeted Nrf2 sequence. (H) Dual-luciferase reporter assay for Nrf2. (I) Immunofluorescence analysis of Nrf2 and Sirt2 expression. ${ }^{* *} \mathrm{P}<0.01$ vs. negative control group. miR, microRNA; Nrf2, nuclear factor erythroid 2-related factor 2; Sirt2, sirtuin 2; wt, wild-type; mut, mutant; STAT, signal transducer and activator of transcription; PI3K, phosphoinositide 3-kinase; TGF- $\beta 1$, transforming growth factor $\beta 1$; TLR4, Toll-like receptor 4; TGR5, Takeda G-protein-coupled receptor 5; NF- $\mathrm{B}$, nuclear factor $\kappa \mathrm{B}$; MyD88, myeloid differentiation primary response 88; HIF-1 $\alpha$, hypoxia-inducible factor $1 \alpha$; c-JNK, c-Jun N-terminal kinase; ERK, extracellular-signal-regulated kinase.

cat. nos. sc-362262 and sc-362272; Santa Cruz Biotechnology, Inc., Dallas, TX, USA) at $37^{\circ} \mathrm{C}$ for $1 \mathrm{~h}$ and stained with DAPI for $30 \mathrm{~min}$ in darkness. Images of cells were captured under fluorescence microscopy.

Statistical analysis. Results are expressed as the mean \pm standard deviation. Student's t-test was performed for statistical significance between two groups. One-way analysis of variance and Tukey's post hoc test were performed for parametric multivariable analysis.

\section{Results}

miR-140-5p expression in mice with AS. As presented in Fig. 1A, H\&E staining indicated plaques in mice with AS, compared with the control group. Furthermore, the MDA 

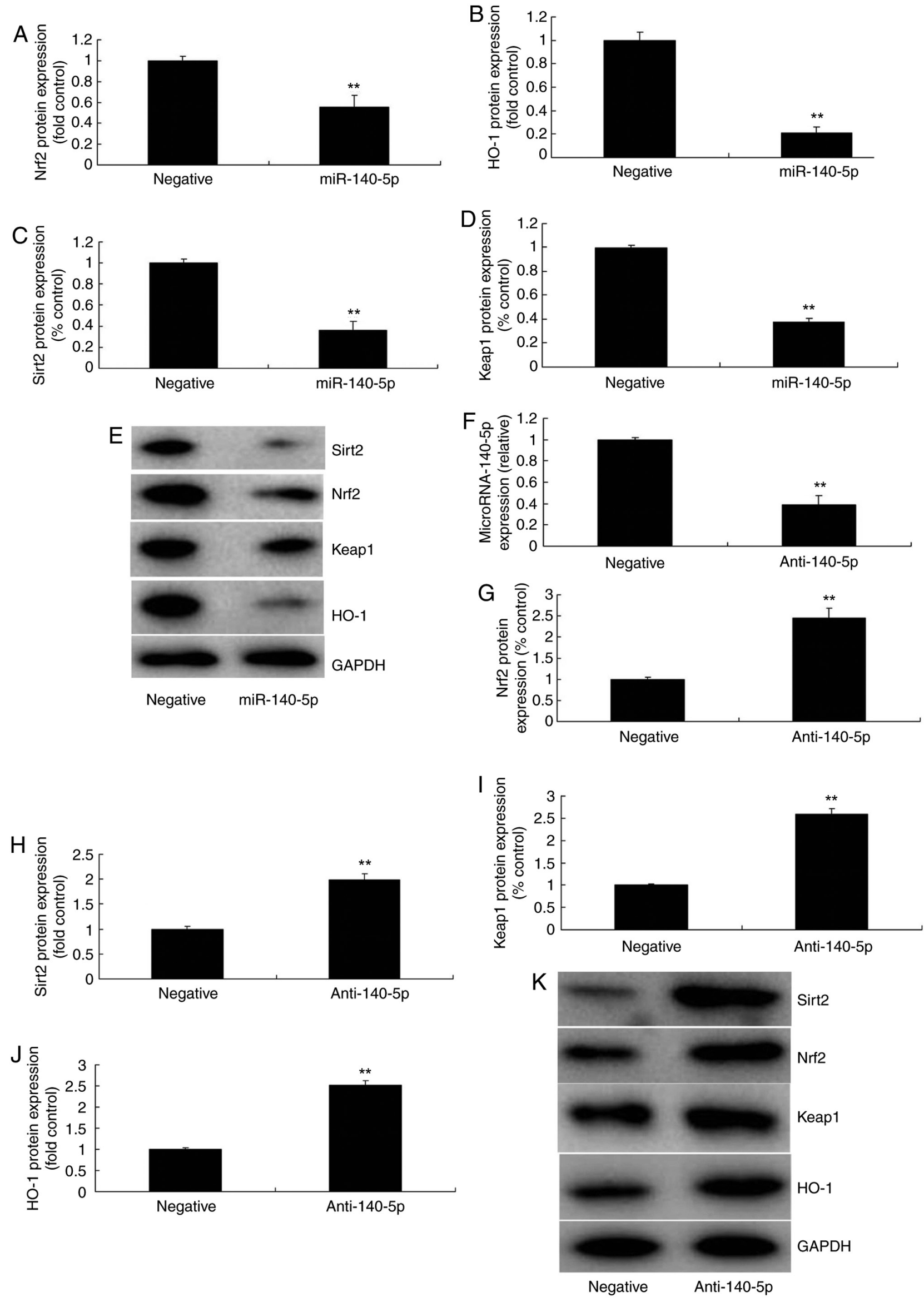

Figure 3. miR-140-5p regulates Nrf2, Sirt2, Keap1 and HO-1 expression. (A) Nrf2, (B) HO-1, (C) Sirt2 and (D) Keap1 protein expression as assessed by (E) western blotting following overexpression of miR-140-5p. (F) Quantitative polymerase chain reaction analysis of miR-140-5p. (G) Nrf2, (H) Sirt2, (I) Keap1 and $(\mathrm{J}) \mathrm{HO}-1$ protein expression as assessed by $(\mathrm{K})$ western blotting following downregulation of miR-140-5p. ${ }^{* *} \mathrm{P}<0.01$ vs. negative group. miR, microRNA; Nrf2, nuclear factor erythroid 2-related factor 2; Sirt2, sirtuin 2; Keap1, Kelch-like enoyl-CoA hydratase-associated protein 1; HO-1, heme oxygenase 1. 

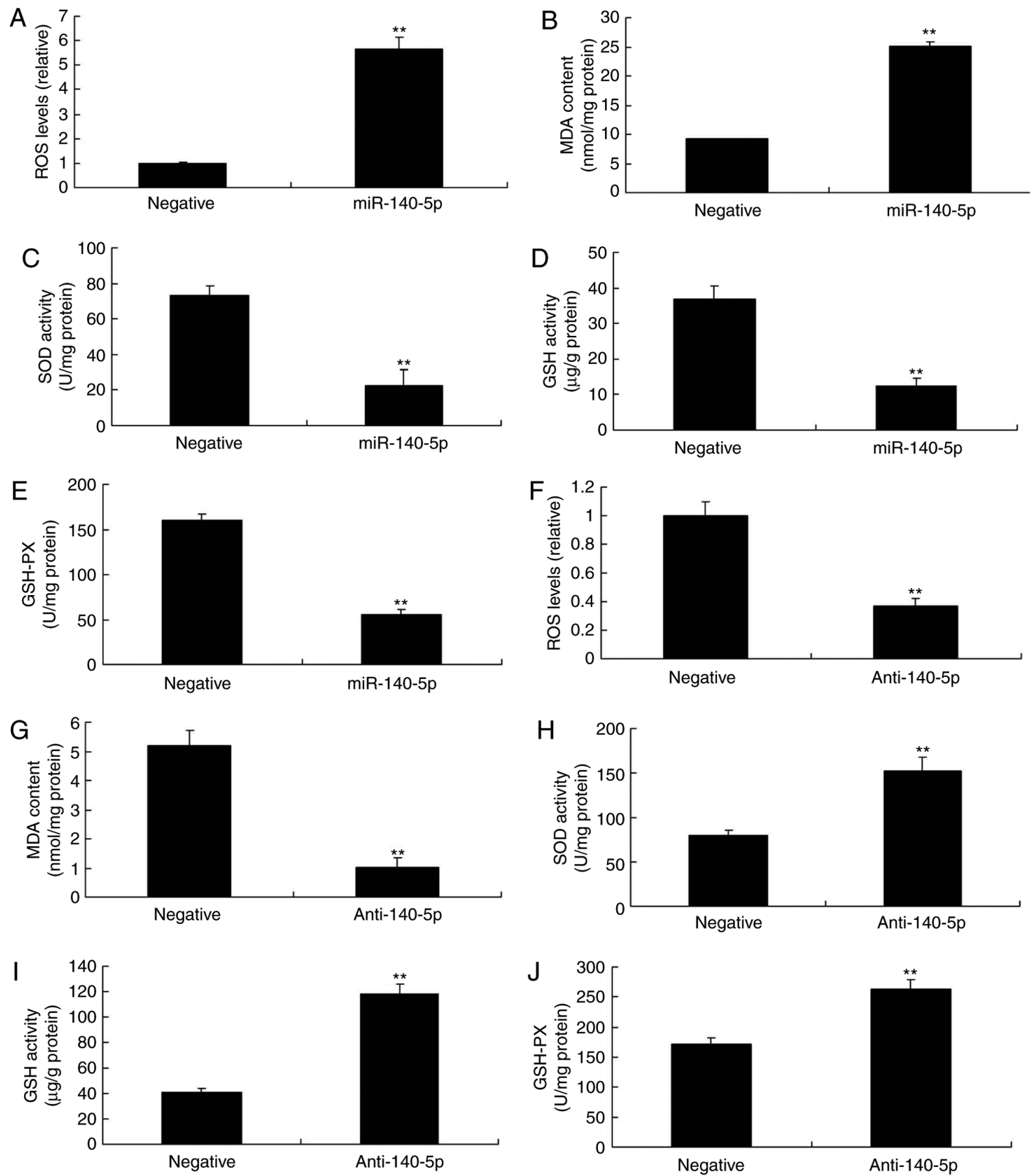

Figure 4. miR-140-5p regulates oxidative stress in an in vitro model. (A) ROS, (B) MDA, (C) SOD, (D) GSH and (E) GSH-PX levels following overexpression of miR-140-5p. (F) ROS, (G) MDA, (H) SOD, (I) GSH and (J) GSH-Px levels following downregulation of miR-140-5p. ${ }^{* *} \mathrm{P}<0.01$ vs. negative control group. miR, microRNA; ROS, reactive oxygen species; MDA, malondialdehyde; SOD, superoxide dismutase; GSH, glutathione; GSH-Px, glutathione peroxidase.

level was increased, whereas the levels of SOD, GSH and GSH-Px were decreased, in mice with AS, compared with the control group (Fig. 1B-E, respectively). A microarray assay was used to analyze the alterations in miR-140-5p expression in mice with AS. As presented in Fig. $1 F$ and G, miR-140-5p expression was increased in mice with AS, compared with the control. Thus, miR-140-5p expression may serve an important function in AS.

miR-140-5p regulates the expression of Nrf2 and Sirt2. A microarray assay was utilized to investigate the underlying molecular mechanism of miR-140-5p in AS. In brief, miR-140-5p mimic was used to increase the expression of miR-140-5p in vitro in mice with AS, compared with negative control group (Fig. 2A). A microarray assay and qPCR indicated that the expression of $\mathrm{Nrf} 2$ and Sirt 2 was inhibited in vitro by overexpression of miR-140-5p, compared with the negative control group (Fig. 2B-D). A dual-luciferase reporter assay indicated that miR-140-5p targeted Nrf2 and Sirt2 expression (Fig. 2E-H). In addition, an immunofluorescence assay indicated that overexpression of miR-140-5p suppressed the expression of $\mathrm{Nrf} 2$ and Sirt 2 in the in vitro model of AS, in 

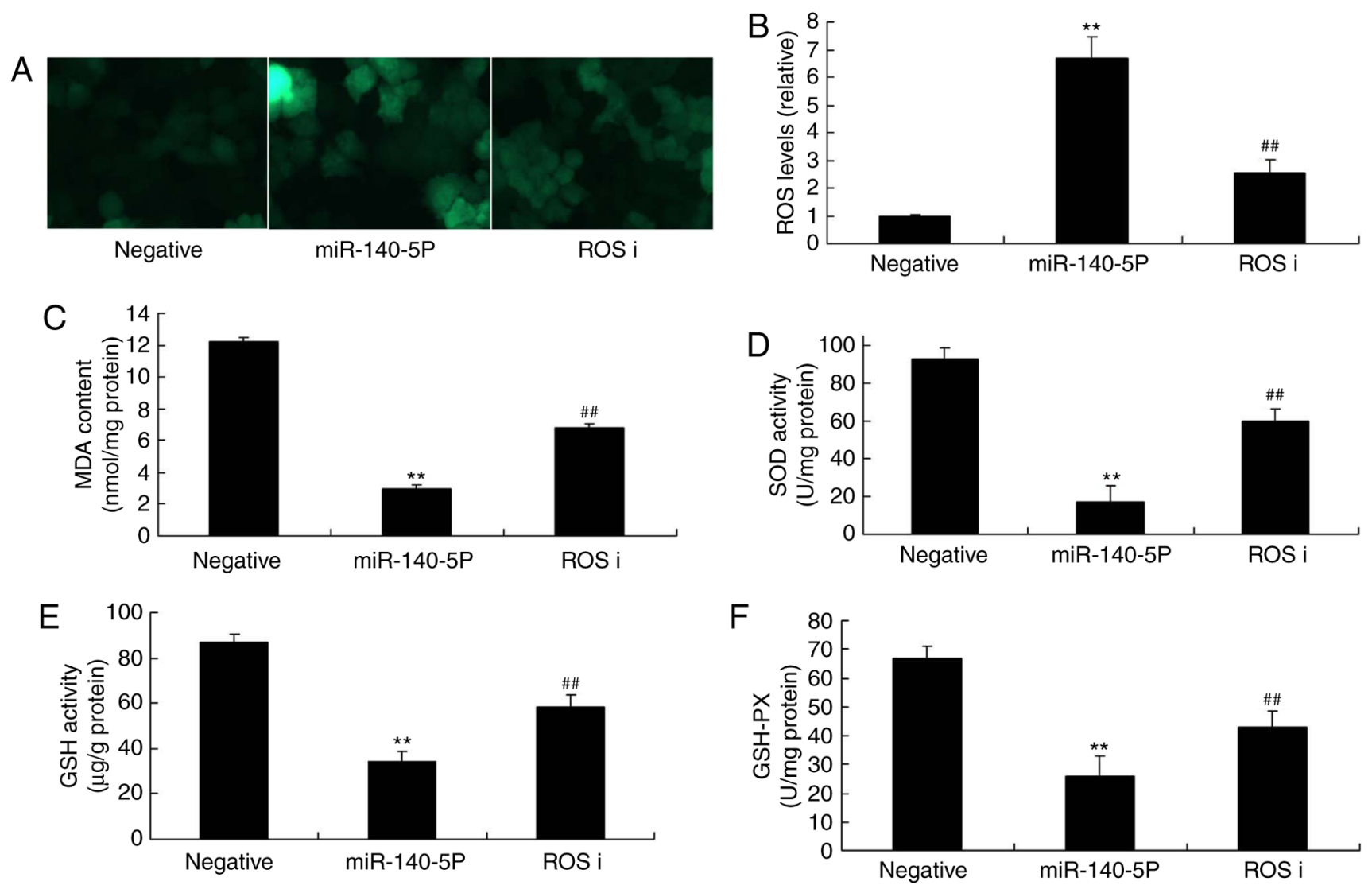

Figure 5. Effect of ROS inhibitor on miR-140-5p in the oxidative stress in vitro model. (A) ROS fluorescence. (B) ROS, (C) MDA, (D) SOD, (E) GSH and (F) GSH-PX levels. ROS inhibitor was added to cells overexpressing miR-140-5p. ROS, reactive oxygen species; miR, microRNA; MDA, malondialdehyde; SOD, superoxide dismutase; GSH, glutathione; GSH-PX, glutathione peroxidase; ROS i, ROS inhibitor. ${ }^{* *} \mathrm{P}<0.01$ vs. negative control group, ${ }^{\# *} \mathrm{P}<0.01$ vs. miR-140-5p overexpression group.

comparison with the negative control group (Fig. 2I). Western blot analysis indicated that overexpression of miR-140-5p suppressed the protein expression of Nrf2, Sirt2, Keap1 and HO-1 in the in vitro model of AS, compared with the negative control group (Fig. 3A-E). Furthermore, anti-miR-140-5p mimic was further employed to decrease the expression of miR-140-5p in the in vitro model of AS, compared with the negative control group (Fig. 3F). However, downregulation of miR-140-5p induced the protein expression of Nrf2, Sirt2, Keap1 and HO-1 in the in vitro model of AS, compared with the negative control group (Fig. 3G-K).

miR-140-5p regulates oxidative stress in vitro. To investigate the function of miR-140-5p in vitro, the alterations in oxidative stress following miR-140-5p transfection in HUVECs were investigated. As presented in Fig. 4A-E, overexpression of miR-140-5p led to increased ROS and MDA levels, whereas it led to decreased levels of SOD, GSH and GSH-Px in vitro, compared with negative group. Downregulation of miR-140-5p decreased the levels of ROS and MDA, and increased those of SOD, GSH and GSH-Px in vitro, compared with negative group (Fig. 4F-J). These results indicated that miR-140-5p regulated Nrf2 and Sirt2 expression to affect oxidative stress in vitro.

ROS inhibitor inhibits the effects of miR-140-5p on oxidative stress in vitro. To investigate the function of ROS on the effects of miR-140-5p on oxidative stress in vitro,
ROS inhibitor was used to decrease the ROS levels in the in vitro model of miR-140-5p overexpression, compared with the miR-140-5p overexpression group in the absence of inhibitor (Fig. 5A and B). ROS inhibitor inhibited MDA levels, and increased the levels of SOD, GSH and GSH-Px following overexpression of miR-140-5p, compared with the miR-140-5p overexpression group in the absence of inhibitor (Fig. 5C-F).

Sirt2 agonist or Nrf2 agonist inhibits the effects of miR-140-5p on Nrf2 and Sirt2 expression in vitro. To further determine the function of Sirt2 or Nrf2 in the effects of miR-140-5p on Nrf2 and Sirt2 expression in vitro, Sirt2 agonist or Nrf2 agonist was used to increase the expression of Nrf2 and Sirt2 expression in the in vitro model of miR-140-5p overexpression, compared with miR-140-5p overexpression group in the absence of agonist (Fig. 6).

Sirt 2 agonist or Nrf2 agonist inhibits the effects of miR-140-5p on oxidative stress in vitro. Finally, to determine the function of Sirt 2 or Nrf2 on the effects of miR-140-5p on oxidative stress in vitro, the levels of oxidative stress in vitro following treatment with miR-140-5p and Sirt2 agonist or Nrf2 agonist were determined. As presented in Fig. 7A-E, Sirt2 agonist inhibited the effects of miR-140-5p on increasing the levels of MDA and ROS, and on decreasing the levels of SOD, GSH and GSH-Px in the in vitro model of miR-140-5p overexpression, 

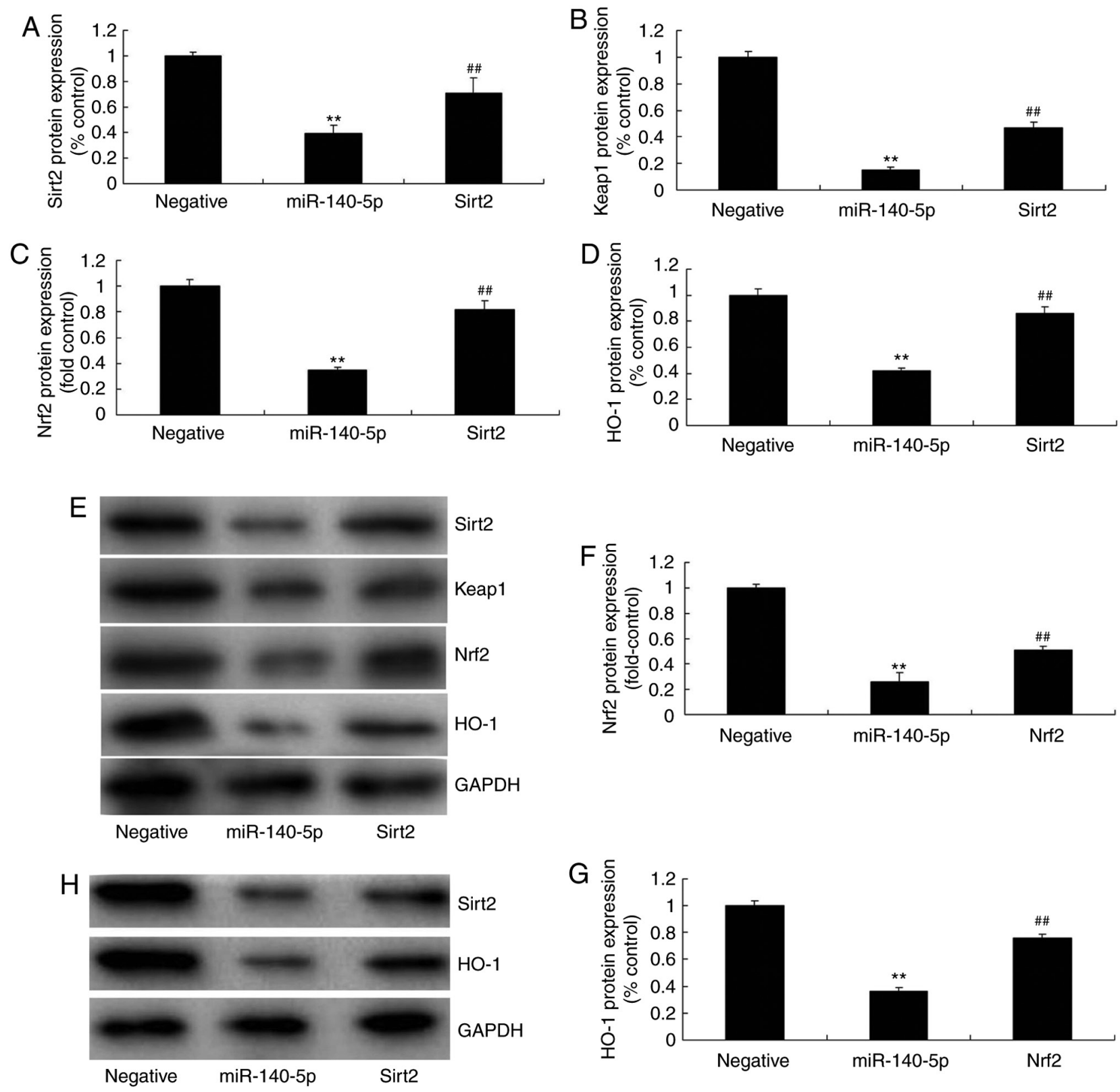

Figure 6. Sirt2 agonist or Nrf2 agonist inhibits the effects of miR-140-5p on Nrf2 and Sirt2 expression in an in vitro model. (A) Sirt2, (B) Keap1, (C) Nrf2 and (D) HO-1 protein expression as assessed by (E) western blotting. (F) Nrf2 and (G) HO-1 protein expression as assessed by (H) western blotting. Agonists were added to cells overexpressing miR-140-5p. ${ }^{* *} \mathrm{P}<0.01$ vs. negative control group. ${ }^{\# / P} \mathrm{P}<0.01$ vs. miR-140-5p overexpression group. Sirt2, sirtuin 2; Nrf2, nuclear factor erythroid 2-related factor 2; miR, microRNA; Keap1, Kelch-like enoyl-CoA hydratase-associated protein 1; HO-1, heme oxygenase 1.

compared with the miR-140-5p overexpression group in the absence of agonist. Sirt2 agonist also decreased the effects of miR-140-5p on increasing the levels of MDA and ROS, and on decreasing the levels of SOD, GSH and GSH-Px in the in vitro model of miR-140-5p overexpression, compared with the miR-140-5p overexpression group in the absence of agonist (Fig. 7F-J). Together, these results indicated that miR-140-5p regulated Nrf2 and Sirt2 expression to affect oxidative stress in vitro.

\section{Discussion}

AS is considered the pathological basis for the occurrence and progression of coronary heart disease (16). miRNAs are a group of endogenous conserved non-coding small-molecule single-stranded RNAs that are widely distributed in eukaryotes and are involved in the regulation of gene expression at the post-transcription level (16). Previous studies have identified that the expression of miRNA is associated with the formation of atherosclerotic plaques $(11,17)$. miRNA serves a function in the regulation of cardiac development as well as multiple cardiac pathophysiological processes such as cardiac hypertrophy, arrhythmia and heart failure $(11,17)$. Certain biomarkers have been identified to be associated with AS (17). However, it is necessary to seek novel biomarkers to provide an earlier, more accurate and sensitive indication of AS $(16,17)$, leading to early clinical diagnosis, treatment and prognosis of patients with AS. Previous studies have demonstrated that the levels of circulating miRNAs vary markedly among various diseases $(16,17)$. As a result, miRNAs may be used 

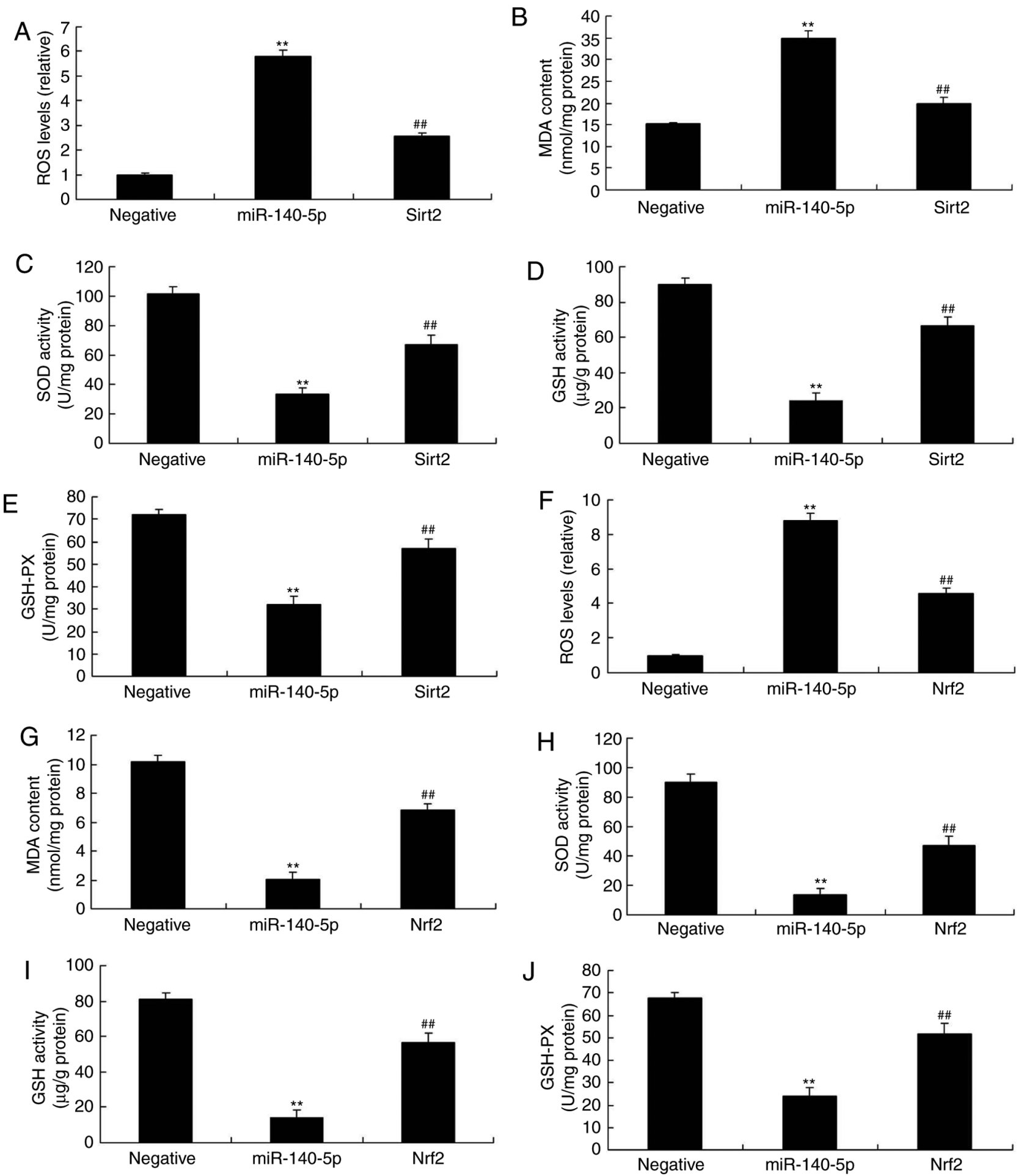

Figure 7. Sirt2 agonist or Nrf2 agonist inhibits the effects of miR-140-5p in an oxidative stress in vitro model. (A) ROS, (B) MDA, (C) SOD, (D) GSH and (E) GSH-Px levels following treatment with a Sirt2 agonist. (F) ROS, (G) MDA, (H) SOD, (I) GSH and (J) GSH-Px levels following treatment with a Nrf2 agonist. Agonists were added to cells overexpressing miR-140-5p." $\mathrm{P}<0.01$ vs. negative control group. ${ }^{\# \#} \mathrm{P}<0.01$ vs. miR-140-5p overexpression group. Sirt2, sirtuin 2; Nrf2, nuclear factor erythroid 2-related factor 2; miR, microRNA; ROS, reactive oxygen species; MDA, malondialdehyde; SOD, superoxide dismutase; GSH, glutathione; GSH-Px, glutathione peroxidase.

as biomarkers of diseases or be used to evaluate the severity and prognosis of disease. In the present study, it was identified that miR-140-5p expression was also increased in mice with AS. Zhao et al (18) identified that miR-140-5p was induced in doxorubicin-induced cardiotoxicity, therefore miR-140-5p expression may be an important element in AS.
Oxidative stress levels can be detected by determining antioxidant enzyme system levels, non-enzymatic antioxidant levels and oxidative product levels (3). In patients with pre-diabetes accompanied by a highly oxidative stress state, the generation of free radicals increases in the body with the aggravation of glucose metabolism disorders (19). This will 


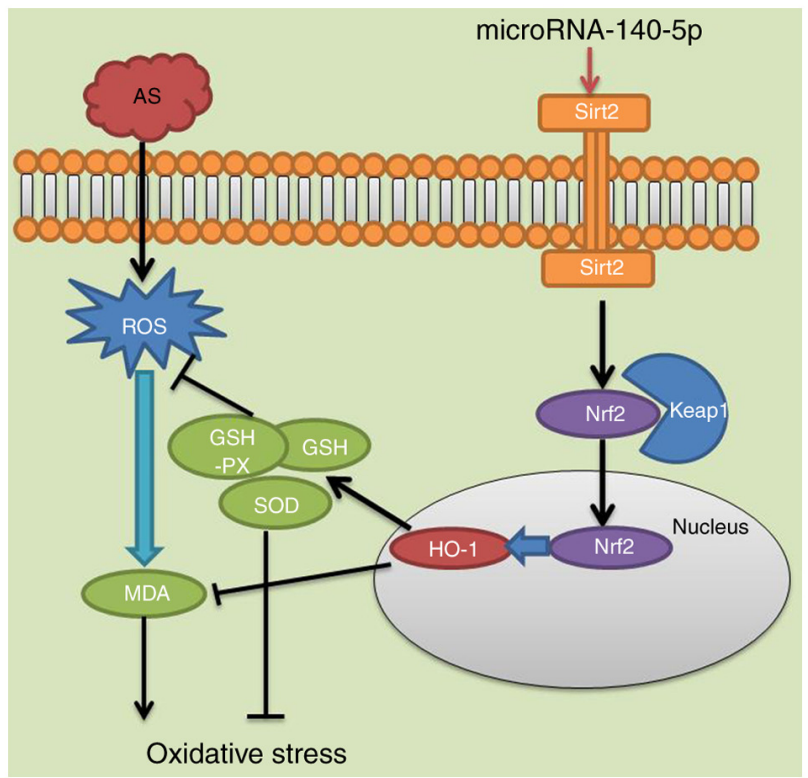

Figure 8. MicroRNA-140-5p aggravates hypertension and oxidative stress of atherosclerosis via targeting Nrf2 and Sirt2. Nrf2, nuclear factor erythroid 2-related factor 2; Sirt2, sirtuin 2; AS, atherosclerosis; ROS, reactive oxygen species; MDA, malondialdehyde; GSH-PX, glutathione peroxidase; GSH glutathione; SOD, superoxide dismutase; HO-1, heme oxygenase 1; keap1, Kelch-like enoyl-CoA hydratase-associated protein 1

lead to decreased antioxidant capacity, increased levels of oxidative stress and ultimately imbalance of oxidation and antioxidation (20). The occurrence and progression of AS are associated with oxidative stress (19). Oxidative stress already exists in patients with AS, accompanied by a weakened antioxidant capacity. In patients with AS combined with diabetes, the degree of oxidative damage gradually increases as the disease progresses (19). It was identified that overexpression of miR-140-5p increased ROS and MDA levels, and decreased SOD, GSH and GSH-Px levels, in the in vitro model of AS. The ROS inhibitor decreased the effects of miR-140-5p on oxidative stress in the in vitro model of AS.

The primary physiological characteristic of Nrf2 is its high sensitivity to oxidative stress and exogenous toxicants in the body (21). In the process of body defense, Nrf2 serves a critical function in the oxidative stress induced by exogenous toxicants. Keap1 is one of the major receptors of Nrf2; all of its cysteine residues are able to interact with the HO-1 inducer (5). This inducer interacts with specific residues on Keap1 and causes conformational changes in Keap1, which are able to trigger Nrf2 dissociation from Keap1 (21). Thus, alterations in its structure affect the complete expression of $\mathrm{Nrf2}$, i.e. the nuclear export sequence in Keap1 is essential to inhibit binding of the antioxidant-response element (ARE) HO-1 with Nrf2 (21). In the present study, it was identified that overexpression of miR-140-5p suppressed Nrf2, Sirt2, Keap1 and HO-1 protein expression in the in vitro model of AS. Liao et al (22) identified that miR-140-5p attenuated oxidative stress in cisplatin-induced acute kidney injury through the Nrf2/ARE signaling pathway.

Sirt2 serves an important function in oxidative stress and autophagy in endothelial cells (23). The specific regulatory mechanism of Sirt2 on vascular endothelial cells remains unknown; however, it has been identified that Sirt2 may be involved in the mediation of endothelial cell migration induced by angiotensin II by regulating the acetylation of $\alpha$-tubulin and microtubule recombination (23). cDNA microarrays were used to confirm that Sirt2 was knocked down in primary cultured endothelial cells, which would lead to changes in general gene expression during oxidative stress (9). Among them, the majority were not altered following Sirt1 knockdown (9). Of note, drug inhibition of Sirt2 was able to decrease oxidative stress-induced endothelial cell injury (10). In the present study, it was identified that Sirt2 agonist or Nrf2 agonist inhibited the effects of miR-140-5p on oxidative stress in an in vitro model. Zhao et al (18) identified that miR-140-5p aggravates doxorubicin-induced cardiotoxicity by targeting Nrf2 and Sirt2. miR-140-5p regulates ROS levels and the ROS downstream signaling pathway is a focus of further study, including nuclear factor $\mathrm{\kappa B}$ and cryopyrin.

In conclusion, in the present study, the potential effects and underlying molecular mechanisms of miR-140-5p-aggravated hypertension and oxidative stress of patients with AS by targeting Nrf2 and Sirt2 were investigated. It was identified that miR-140-5p acts as an excellent antioxidant and induced the Sirt2/Nrf2/HO-1 signaling pathway to suppress oxidative stress in AS (Fig. 8). Therefore, the results of the present study suggest that miR-140-5p/Sirt2/Nrf2 have an important function in the prevention and treatment of AS.

\section{Acknowledgements}

Not applicable.

\section{Funding}

No funding was received.

\section{Availability of data and materials}

The analyzed data sets generated during the study are available from the corresponding author on reasonable request.

\section{Authors' contributions}

XHY designed the experiments; QQL, KR, SHL, WML and CJH performed the experiments; XHY analyzed the data; $\mathrm{XHY}$ wrote the manuscript.

\section{Ethics approval and consent to participate}

Animal experiments were approved by the Ethics Committee of The Ninth People's Hospital (Shanghai Jiaotong University School of Medicine, Shanghai, China).

\section{Patient consent for publication}

Not applicable.

\section{Competing interests}

The authors declare that they have no competing interests. 


\section{References}

1. Sirotina S, Ponomarenko I, Kharchenko A, Bykanova M, Bocharova A, Vagaytseva K, Stepanov V, Churnosov M, Solodilova M and Polonikov A: A Novel polymorphism in the promoter of the CYP4A11 gene is associated with susceptibility to coronary artery disease. Dis Markers 2018: 5812802, 2018.

2. Kiris T, Avci E and Çelik A: Combined value of left ventricular ejection fraction and the Model for End-Stage Liver Disease (MELD) score for predicting mortality in patients with acute coronary syndrome who were undergoing percutaneous coronary intervention. BMC Cardiovasc Disord 18: 44, 2018.

3. Shahid SU, Shabana and Humphries S: The SNP rs10911021 is associated with oxidative stress in coronary heart disease patients from Pakistan. Lipids Health Dis 17: 6, 2018.

4. Ishikawa $\mathrm{T}$ and Seki $\mathrm{K}$ : The association between oxidative stress and endothelial dysfunction in early childhood patients with Kawasaki disease. BMC Cardiovasc Disord 18: 30, 2018.

5. Yu H, Shi L, Zhao S, Sun Y, Gao Y, Sun Y and Qi G: Triptolide attenuates myocardial ischemia/reperfusion injuries in rats by inducing the activation of $\mathrm{Nrf} 2 / \mathrm{HO}-1$ defense pathway. Cardiovasc Toxicol 16: 325-335, 2016.

6. Donovan EL, McCord JM, Reuland DJ, Miller BF and Hamilton KL: Phytochemical activation of Nrf2 protects human coronary artery endothelial cells against an oxidative challenge. Oxid Med Cell Longev 2012: 132931, 2012.

7. Siow RC, Ishii T and Mann GE: Modulation of antioxidant gene expression by 4-hydroxynonenal: atheroprotective role of the Nrf2/ARE transcription pathway. Redox Rep 12: 11-15, 2007.

8. Tang X, Chen XF, Wang NY, Wang XM, Liang ST, Zheng W, $\mathrm{Lu}$ YB, Zhao X, Hao DL, Zhang ZQ, et al: SIRT2 Acts as a cardioprotective deacetylase in pathological cardiac hypertrophy. Circulation 136: 2051-2067, 2017.

9. Matsushima S and Sadoshima J: The role of sirtuins in cardiac disease. Am J Physiol Heart Circ Physiol 309: H1375-H1389, 2015.

10. Yang W, Gao F, Zhang P, Pang S, Cui Y, Liu L, Wei G and Yan B: Functional genetic variants within the SIRT2 gene promoter in acute myocardial infarction. PLoS One 12: e0176245, 2017.

11. Nanoudis S, Pikilidou M, Yavropoulou M and Zebekakis P: The role of MicroRNAs in arterial stiffness and arterial calcification. An update and review of the literature. Front Genet 8: 209, 2017.

12. Li S, Lee C, Song J, Lu C, Liu J, Cui Y, Liang H, Cao C, Zhang F and Chen H: Circulating microRNAs as potential biomarkers for coronary plaque rupture. Oncotarget 8: 48145-48156, 2017.

13. Jansen F, Schäfer L, Wang H, Schmitz T, Flender A, Schueler R, Hammerstingl C, Nickenig G, Sinning JM and Werner N: Kinetics of circulating MicroRNAs in response to cardiac stress in patients with coronary artery disease. J Am Heart Assoc 6 : pii: e005270, 2017.

14. Li XD, Yang YJ, Wang LY, Qiao SB, Lu XF, Wu YJ, Xu B, Li HF and Gu DF: Elevated plasma miRNA-122, -140-3p, -720, -2861, and -3149 during early period of acute coronary syndrome are derived from peripheral blood mononuclear cells. PLoS One 12 : e0184256, 2017.
15. Livak KJ and Schmittgen TD: Analysis of relative gene expression data using real-time quantitative PCR and the 2(-Delta Delta $\mathrm{C}(\mathrm{T})$ ) method. Methods 25: 402-408, 2001.

16. Adams A, Bojara W and Schunk K: Early diagnosis and treatment of coronary heart disease in asymptomatic subjects with advanced vascular atherosclerosis of the carotid artery (Type III and IV b Findings Using Ultrasound) and risk factors. Cardiol Res 9: 22-27, 2018

17. Wu R, Shen D, Sohun H, Ge D, Chen X, Wang X, Chen R, Wu Y, Zeng J, Rong X, et al: miR-186, a serum microRNA, induces endothelial cell apoptosis by targeting SMAD6 in Kawasaki disease. Int J Mol Med 41: 1899-1908, 2018.

18. Zhao L, Qi Y, Xu L, Tao X, Han X, Yin L and Peng J: MicroRNA-140-5p aggravates doxorubicin-induced cardiotoxicity by promoting myocardial oxidative stress via targeting Nrf2 and Sirt2. Redox Biol 15: 284-296, 2018.

19. Tanabe K, Kawai Y, Kitayama M, Akao H, Ishida R, Motoyama A, Wakasa M, Saito R, Aoki H, Fujibayashi K, et al: Increased levels of the oxidative stress marker, nitrotyrosine in patients with provocation test-induced coronary vasospasm. J Cardiol 64: 86-90, 2014.

20. Kim JY, Lee JW, Youn YJ, Ahn MS, Ahn SG, Yoo BS, Lee SH, Yoon J and Choe KH: Urinary levels of 8-iso-prostaglandin f2 $\alpha$ and 8-hydroxydeoxyguanine as markers of oxidative stress in patients with coronary artery disease. Korean Circ J 42: 614-617, 2012.

21. Donnarumma E, Bhushan S, Bradley JM, Otsuka H, Donnelly EL, Lefer DJ and Islam KN: Nitrite Therapy ameliorates myocardial dysfunction via $\mathrm{H} 2 \mathrm{~S}$ and nuclear factor-erythroid 2-related factor 2 (Nrf2)-dependent signaling in chronic heart failure. J Am Heart Assoc 5: pii: e003551, 2016.

22. Liao W, Fu Z, Zou Y, Wen D, Ma H, Zhou F, Chen Y, Zhang M and Zhang W: MicroRNA-140-5p attenuated oxidative stress in Cisplatin induced acute kidney injury by activating Nrf2/ARE athway through a Keap1-independent mechanism. Exp Cell Res 360: 292-302, 2017.

23. Turdi S, Li Q, Lopez FL and Ren J: Catalase alleviates cardiomyocyte dysfunction in diabetes: Role of Akt, Forkhead transcriptional factor and silent information regulator 2. Life Sci 81: 895-905, 2007

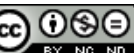

This work is licensed under a Creative Commons Attribution-NonCommercial-NoDerivatives 4.0 International (CC BY-NC-ND 4.0) License. 